\title{
Utilidad de la Clínica Obstétrica para el Manejo del antecedente de Cesárea
}

\author{
Victor Rodríguez; Javier Ardila; Luis F. Botero*
}

En las últimas décadas se ha presentado un fenómeno francamente preocupante, relacionado con el aumento escandaloso de la tasa de cesáreas con implicaciones importantes como el aumento en los costos, implicaciones éticas y de morbimortalidad relacionada con la intervención. Se han realizado múltiples estudios al respecto y se ha concluido que una de las estrategias más efectivas para disminuir la tasa de cesáreas es la de permitir parto vaginal a las pacientes con antecedente de cesárea después de una adecuada selección que asegure la disminución de complicaciones como la ruptura uterina.

Conscientes de nuestro compromiso como departamento universitario en el entrenamiento de personal médico para contribuir a solucionar los problemas de la especialidad, fué como proyectamos la realización de este trabajo, en el que se capturó una muestra de 390 pacientes con cesárea previa que tuvieron su control prenatal en el ISS y fueron remitidas al término de su embarazo para su atención de parto. Estas fueron examinadas y clasificadas como aptas para prueba de trabajo de parto o programadas para la cesárea iterativa electiva, por un equipo de trabajo fijo conformado por el coordinador y el jefe de la unidad obstétrica. A las pacientes aptas se les presentó detalladamente las ventajas y riesgos de las dos opciones cesárea o parto vaginal y se respetó la voluntad de la paciente, de tal forma que a 135 (35\%) se les dió prueba de trabajo y de estas $75(56 \%)$ tuvieron parto por vía vaginal mientras que a las $60(44 \%)$ restantes se les practicó cesárea intraparto.

No se presentó ninguna muerte materna ni fetal. Hubo un caso de ruptura uterina diagnosticada de la revisión uterina post-parto, que se manejó sin dificultad.

Recomendamos el trabajo de parto en el antecedente de cesárea cuando se cumplen todas las condiciones necesarias.

PALABRAS ClAVES: Parto por Cesárea, Parto Vaginal, Clínica Obstetrica, Antecedente de cesárea.

In the last decades a phenomenon has been presented preocupante frankly, related with the scandalous increase of the rate of Caesarean with important implications as the increase in the costs, ethical implications and of morbimortalidad related with the intervention. They have been carried out multiple studies in this respect and it has been concluded that one of the most effective strategies to diminish the rate of Caesarean operations is the one of allowing vaginal childbirth to the patients with Caesarean operation antecedent after an appropriate selection that assures the decrease of complications like the uterine rupture.

Aware of our commitment like university department in medical personnel's training to contribute to solve the problems of the specialty, fué like we project the realization of this work, in which a sample of 390 patients was captured with previous Caesarean operation that you/ they had its prenatal control in the ISS and they were remitted at the end of its pregnancy for its childbirth attention. These were examined and classified as capable for test of childbirth work or programmed for the Caesarean elective iterativa, for a team of fixed work conformed by the coordinator and the boss of the obstetric unit. To the capable patients they were presented the advantages and risks of the Caesarean two options or vaginal childbirth detailedly and the patient's will was respected, in such a way that at 135 (35\%) you them dió proves of work and of 75 o'clock $(56 \%)$ they had childbirth for via vaginal while at 60 o'clock (44\%) remaining they were practiced Caesarean intraparto.

Any maternal neither fetal death was not presented. There was a case of diagnosed uterine rupture of the revision uterine post-childbirth that was managed without difficulty.

We recommend the childbirth work in the Caesarean operation antecedent when all the necessary conditions are completed.

KEY WORDS: Cesarean delivery, Vaginal delivery, Obstetric Clinic, Previus cesarean.

En la evolución de la cesárea como procedimiento se observa que en su inicio se realizó en situaciones que ponían en riesgo la vida de la paciente y en aquellos casos en que era físicamente imposible la atención del parto por vía vaginal con una muy alta tasa de morbi-mortalidad

\footnotetext{
* Departamento de Ginecología y Obstetricia, Hospital San
} Ignacio, Universidad Javeriana materna y perinatal. Posteriormente, con los notables progresos en asepsia y antisepsia, mejoría de la infraestructura hospitalaria y depuración de la técnica quirúrgica, las complicaciones se disminuyeron considerablemente pero aún en tales condiciones de idoneidad estas continuan siendo más altas que las que se presentan con el parto eutócico. Por otro lado, el surgimiento de tecnologías como la monitoría fetal y la ecografía obstétrica contribuyen a disminuir la morbi-mortalidad neonatal 
a costa del aumento de la tasa de cesáreas. Esta situación trae como consecuencia la sobreestimación de la cesárea como procedimiento de elección en muchas situaciones clínicas que con un análisis más juicioso y un manejo más claramente dirigido pueden terminar en un parto vaginal.

La racionalización de los recursos guardando el justo equilibrio con el ofrecimiento de una medicina de alta calidad ha sido el principal motor para planear y desarrollar estrategias que busquen disminuir las tasas de cesáreas en pacientes con antecedente de cesárea.

Con lo expuesto anteriormente y además teniendo en cuenta nuestro compromiso como Hospital Universitario de contribuir con experiencias propias a los requerimientos del país en lo que compete a nuestra área propusimos unos objetivos muy claros en la Clínica Obstétrica tales como seleccionar adecuadamente a las pacientes con antecedente de cesárea con una gran posibilidad de tener un parto por vía vaginal teniendo en cuenta la historia clínica, las razones que propiciaron la anterior cesárea y desde luego la valoración clínica de la pelvis. Por otro lado, es fundamental describir las causas más frecuentemente encontradas entre las pacientes por las cuales no permiten la prueba de trabajo de parto.

\section{Materiales y Métodos}

Se constituyó un equipo de trabajo dentro del cual se estableció desde el inicio una estandarización de conceptos que permitieran asegurar que las pacientes estuvieran siendo valoradas siempre bajo la misma óptica, en lo concerniente a:

- Historia clínica

- Datos confiables de edad gestacional (fecha de última regla, ecografía seriada)

- Evolución de el embarazo actual

- Condiciones que motivaron la cesárea previa

- Condiciones del post-operatorio y complicaciones

- Pelvimetria interna

- Características del cuello uterino

- Relación presentación-pelvis ósea

Para estos efectos se realizó un instrumento de recolección que evitaba perder información y además facilitaba el posterior análisis de la información.

Una vez se logró recoger la información pertinente en cada paciente, se clasificaron en dos grupos; apta para prueba de trabajo de parto y no apta para prueba de trabajo de parto.

A las pacientes que se les consideró aptas, se les planteó esta alternativa informándoles los riesgos y ventajas, aclarando oportunamente las dudas que surgieran y finalmente la conducta se tomó siempre respetando la decisión efectuada por la paciente con todos los elementos de juicio que se le daban durante la entrevista. El trabajo de parto de estas pacientes incluyó monitoría fetal intermitente, analgesia peridural para la fase activa, libertad absoluta al obstetra para instrumentar profilácticamente o no el parto y revisión manual en todas las pacientes. El uso de Oxitocina a dosis bajas como refuerzo del trabajo de parto también se dejó a criterio del médico involucrado en la atención.
A las pacientes que se les consideró no aptas, se les programó en la respectiva junta para la cesárea y se les dió las indicaciones necesarias.

Los criterios de exclusión para prueba de trabajo de parto fueron:

1. No aceptación de la paciente

2. Cesárea corporal previa.

3. Miomectomía previa

4. Distocias de presentación

5. Retardo del crecimiento intrauterino

6. Insuficiencia placentaria

7. Complicaciones obstétricas que se benefi cien con la cesárea

8. Antecedente de endometritis post-cesárea

9. Antecedente de dos o más cesáreas

10. Pacientes que fueron operadas en institucio nes que no cumplian con los requisitos de recursos y buen nivel científico.

11. Embarazo gemelar

12. Pacientes que requirieron inducción del tra bajo de parto por cualquier razón médica o por embarazo prolongado.

13. Placentación anormal.

14. Cirugía vaginal previa

La muestra del estudio se tomó de manera consecutiva deacuerdo a los criterios antes expuestos, encontrando la siguiente distribución:

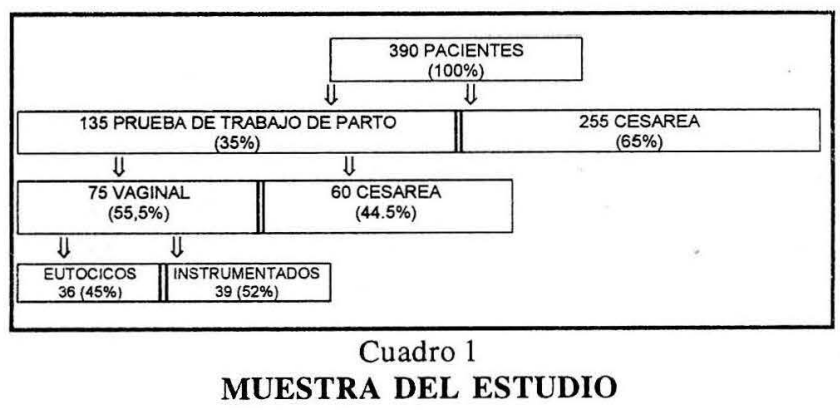

\section{Resultados}

Las razones por las cuales se programaron para cesárea iterativa electiva las pacientes no aptas fueron :

Tabla 1

RAZONES POR LAS CUALES SE CLASIFICARON A LAS PACIENTES COMO NO APTAS PARA PRUEBA DE TRABAJO DE PARTO

\begin{tabular}{|lcl|}
\hline \multicolumn{1}{|c|}{ INDICACION } & \# PACIENTES & $\%$ \\
\hline Antecedente de más de una cesárea & 80 & 31.5 \\
No aceptan prueba de Trabajo de parto & 61 & 24.1 \\
Indicación médica concomitante & 53 & 21 \\
Pelvis no apta & 23 & 9 \\
Presentación anormal & 18 & 7 \\
Antecedente de endometritis & 13 & 5 \\
Antecedente de miomectomía & 3 & 1.2 \\
Enbarazo gemelar & 2 & .8 \\
Placenta previa & 2 & .8 \\
TOTAL & $\mathbf{2 5 5}$ & $\mathbf{1 0 0}$ \\
\hline
\end{tabular}


Podemos observar cuan importante es el Antecedente de más de una cesárea como indicación para nueva cesárea, por lo tanto quisimos profundizar acerca de las causales que motivaron la cesárea anterior, encontrando que definitivamente es un factor determinante para decidir la vía del parto si hay o no cesárea previa (Tabla 2)

Las características generales de la población son las descritas en la (tabla 3)

Dentro de la conducción del trabajo de parto se administró refuerzo con Oxitocina en 8 pacientes que tuvieron el parto por vía vaginal, sin ninguna complicación. (Tabla 4)
Tabla 2

INDICACIONES DE LA CESAREA ANTERIOR

\begin{tabular}{|l|c|}
\hline \multicolumn{1}{|c|}{ INDICACION } & \% \\
\hline & \\
Indicación por cesárea previa & 38.5 \\
Desproporción Céfalo-Pélvica & 26.1 \\
Dilatación estacionaria & 18.7 \\
Sufrimiento Fetal & 6.1 \\
Otras distocias de presentación & 4.9 \\
Placentación anormal & 3.8 \\
Distocia de cuello & 1.8 \\
\hline
\end{tabular}

Tabla 3

CARACTERISTICAS DE LA POBLACION

\begin{tabular}{|l|l|}
\hline \multicolumn{1}{|c|}{ EDAD } & \multicolumn{1}{c|}{ PARIDAD } \\
\hline \multicolumn{1}{|c|}{ Mínima: 17 años } & \multicolumn{1}{c|}{ Máxima: 05 } \\
50\% de las pacientes entre 21-23 años & $74 \%$ de las pacientes entre 2 y 4 partos \\
\hline
\end{tabular}

Tabla 4

TIPO DE PARTO VAGINAL EN PACIENTES QUE SE REFORZARON DURANTE EL TRABAJO DE PARTO

\begin{tabular}{|r|c|}
\hline EUTOCICO & INSTRUMENTADO \\
\hline 2 PACIENTES & 6 PACIENTES \\
\hline $11 \%$ DE LOS & PARTOS VAGINALES \\
\hline
\end{tabular}

Los resultados perinatales en lo concerniente a APGAR, peso y edad gestacional del recién nacido en los grupos parto vaginal eutócico e instrumentado y en el grupo de las cesáreas no presentan mayores diferencias entre sí, como podemos apreciarlo en las tablas 5,6 y 7 .
Tabla 5

APGAR DEL RECIEN NACIDO A LOS CINCO MINUTOS

\begin{tabular}{|lc|cc|cc|}
\hline \multicolumn{2}{|c|}{ EUTOCICO } & \multicolumn{2}{|c|}{ INSTRUMENTADO } & \multicolumn{2}{c|}{ CESAREA } \\
\hline APGAR & $\%$ & APGAR & $\%$ & APGAR & $\%$ \\
Mayorde8 & $100 \%$ & Mayorde8 & $75 \%$ & Mayorde8 & $100 \%$ \\
\hline
\end{tabular}

Las complicaciones maternas fueron escasas en los tres grupos, tal y como lo muestra la Tabla 8 . Con respecto a la ruptura uterina encontrada en una paciente perteneciente al grupo de parto instrumentado se debió fundamentalmente a un periodo expulsivo prolongado ( 3 horas).

Tabla 6

PESO DEL RECIEN NACIDO

\begin{tabular}{|c|c|c|c|c|c|}
\hline \multicolumn{2}{|c|}{ EUTOCICO } & \multicolumn{2}{|c|}{ INSTRUMENTADO } & \multicolumn{2}{|c|}{ CESAREA } \\
\hline PESO & gr. & PESO & gr. & PESO & gr. \\
\hline $\begin{array}{l}\text { Mínimo } \\
\text { Máximo }\end{array}$ & $\begin{array}{l}2440 \\
4000 \\
\end{array}$ & $\begin{array}{l}\text { Mínimo } \\
\text { Máximo }\end{array}$ & $\begin{array}{l}2540 \\
3900 \\
\end{array}$ & $\begin{array}{l}\text { Mínimo } \\
\text { Máximo }\end{array}$ & $\begin{array}{l}2090 \\
4450 \\
\end{array}$ \\
\hline \multicolumn{2}{|c|}{$2500-3500$ gr. el $90 \%$} & \multicolumn{2}{|c|}{$2500-3500$ gr. el $95 \%$} & \multicolumn{2}{|c|}{$2500-3500$ gr. el $90 \%$} \\
\hline
\end{tabular}

Tabla 7

EDAD GESTACIONAL

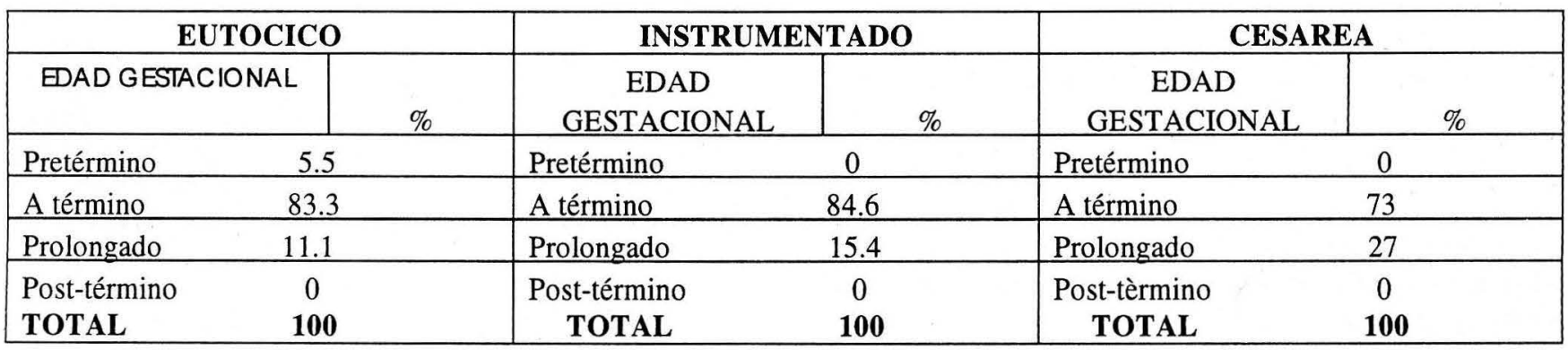


Tabla 8

COMPLICACIONES MATERNAS

\begin{tabular}{|c|c|l|c|l|c|}
\hline \multicolumn{2}{|c|}{ EUTOCICO } & \multicolumn{2}{c|}{ INSTRUMENTADO } & \multicolumn{2}{c|}{ CESAREA } \\
\hline COMPLICACION & No. & \multicolumn{1}{|c|}{ COMPLICACION } & No. & \multicolumn{1}{c|}{ COMPLICACION } & No. \\
\hline Desgarro vaginal & 3 & $\begin{array}{l}\text { Desgaro vaginal } \\
\text { Estancia >48 horas }\end{array}$ & 6 & $\begin{array}{l}\text { Desgarro de segmento } \\
\text { Endometritis }\end{array}$ & 1 \\
& & Ruptura Uterina & 1 & & 1 \\
\hline
\end{tabular}

La solución de continuidad se identificó en la revisión uterina post-parto y se procedió a practicar histerectomía. Es importante enfatizar, que este cuadro no se constituyó en una emergencia dramática y que la recuperación de la paciente fué satisfactoria.

Las pacientes con parto vaginal y con antecedente de cesárea tuvieron las siguientes causales como razón de la cesárea anterior. Tabla 9

Mientras que las pacientes que tuvieron trabajo de parto y terminaron en cesárea, tuvieron las siguientes indicaciones de la cesárea anterior. Tabla 10

Las pacientes sometidas a cesárea ya fuese electiva porque no se les dió prueba de trabajo de parto o aquellas a las cuales se les indicó la cesárea durante la prueba de trabajo de parto, presentaron las siguientes complicaciones. Tabla 11.

\section{COMENTARIOS Y CONCLUSIONES}

La metodología empleada para el estudio permitió realizar una clasificación adecuada de las pacientes de acuerdo a los criterios de exclusión que e expusieron previamente. De la misma manera se dispuso del tiempo necesario para poder hablar con las pacientes que no aceptaban la prueba del trabajo de parto por temor. Este temor era referido específicamente a la mala experiencia previa en lo concerniente a periodos prolongados de trabajos de parto dolorosos y por otra parte a la influencia de los médicos que les habían controlado el actual embarazo. A pesar de esto, el porcentaje de negativa de la paciente a la prueba de trabajo de parto fue del 21.

El antecedente de dos o más cesáreas constituyó la primera causa de exclusión (31.5\%). Este hallazgo demuestra que en nuestro medio es muy frecuente la conducta de "antecedente de cesárea igual a cesárea". Publicaciones recientes han demostrado que el riesgo de estas pacientes no es mucho mayor que el riesgo de las pacientes con una cesárea previa. Esto nos hace pensar que por un lado podríamos empezar a dar prueba de trabajo de parto a pacientes con más de una cesárea previa ; pero lo más importante quizás es que las pacientes con un antecedente de cesárea no deben intervenirse solamente por el hecho de que ya tiene este antecedente. Esto implica desde luego, una ardua labor de convencimiento que persigue un cambio de mentalidad y actitud frente a esta situación.

Las complicaciones médicas y obstétricas incluyen un grupo de pacientes post-término que no iniciaron trabajo de parto espontáneamente y el protocolo no contempló la inclusión de estas pacientes, por lo cual se intervinieron.
Tabla 9

CAUSALES DE LA CESAREA PREVIA EN PACIENTES CON PARTO VAGINA

\begin{tabular}{|l|c|}
\hline \multicolumn{1}{|c|}{ INDICACION } & No. DE \\
PACIENTES
\end{tabular}

Tabla 10

CAUSALES DE PACIENTES SOMETIDAS A TRABAJO DE PARTO Y QUE TERMINARON EN CESAREA

\begin{tabular}{|l|c|}
\hline \multicolumn{1}{|c|}{ INDICACION } & $\begin{array}{c}\text { No. DE } \\
\text { PACIENTES }\end{array}$ \\
\hline $\begin{array}{l}\text { Distocia de prsentación + } \\
\text { Anormalidad placentaria }\end{array}$ & $34(56.5 \%)$ \\
Desproporción Céfalo-pélvica & $13(21.6 \%)$ \\
Médica & $11(18.3 \%)$ \\
Sufrimiento Fetal Agudo & $02(3.3 \%)$ \\
\hline
\end{tabular}

Tabla 11

COMPLICACIONES DE LAS CESAREAS

\begin{tabular}{ccc|}
\hline $\begin{array}{c}\text { POSTERIOR A PRUEBA DE } \\
\text { TRABAJO DE PARTO }\end{array}$ & ELECTIVA \\
\hline $\begin{array}{l}\text { Endometritis } \\
\text { Desgartos }\end{array}$ & $1(1.7 \%)$ & $\begin{array}{c}\text { No hubo } \\
\text { complicaciones de } \\
\text { ninguna naturaleza }\end{array}$ \\
\hline
\end{tabular}

Los resultados obtenidos alcanzando un 55\% de partos vaginales y excelentes resultados perinatales y maternos en todos los grupos (Parto vaginal eutócico, parto vaginal instrumentado y cesárea después de prueba de trabajo de parto y cesárea electiva), podemos afirmar que con el uso juicioso de un grupo de médicos que conforman la Clínica Obstétrica podemos reducir al mínimo los riesgos y de igual manera el índice de cesáreas por antecedente. 
Por otro lado, es importante resaltar que desde el punto de vista económico cada paciente que tenía antecedente de cesárea y que después de la prueba de trabajo tuvo su parto por vía vaginal, presentó un costo que en promedio fue $\$ 150.000$.oo más barato que el de aquellas pacientes a quienes se les practicó la cesárea electiva. Esto sin duda adquiere importancia debido a las circunstancias actuales de nuestras políticas de salud en las que el concepto de costo-efectividad debe respaldar siempre las diferentes alternativas de tratamiento.

\section{BIBLIOGRAFIA}

1. Benedetti Tj, Platt L, Druzin M. Vaginal delivery after previous cesarean section for a non recurrent cause. Am.J. Obstet. Gynecol, $142: 358,1982$

2. Bottoms s.f, roses me, sukolry. The increase in the cesarean birth rate. N.Engl. J. Med $302: 559,1980$

3. Gibss Ce. Planed vaginal delivery following cesarean section. Clin.Obstet. Gynecol. $23: 507,1980$

4. Lavin Jp, Stephens Rj, Miodovnik M, Banden Tp. Vaginal delivery in patients with a prior cesarean section. Obstet. And Gynecol. $59: 135,1982$

5. Meir Pr, Pomeco Rp. Trial of labor following cesarean section. Alow year experience. Am.J.Obstet and Gynecol. 144 :671. 1982

6. O'Driscoll R. Correlation of decrease in perinatal mortality and increasen section rate. Obstet. Gynecol. $61: 1.193$

7. Sachs Bp, Mc.canty Bj., Rubin G., Burton A., Terry J., Tyler Cw Jr. Cesarean section: Risk and benefits for mother ans fetus. JAMA $250: 21 \quad 57,1983$

8. Mann D, Vaginal Birth after C-Section. N. Engl. J. Med 335:689695, 1996

9. Chazotte C; Madden R; Cohen Wr. Labor patterns in women with previous cesareans. Obstet Gynecol, 1990 Mar, 75:3 Pt 1, 350-5

10. Nguyen Tv; Dinh Tv; Suresh Ms; Kinch Ra; Anderson GD. Vaginal birth after cesarean section at the University of Texas. J Reprod Med, 1992 Oct, 37:10, 880-2

11. Van Roosmalen J. Vaginal birth after cesarean section in rural Tanzania. Int J Gynaecol Obstet, 1991 Mar, 34:3, 211-5

12.- Rosen Mg; Dickinson Jc; Westhoff Cl. Vaginal birth after cesarean: a meta-analysis of morbidity and mortality. Obstet Gynecol, 1991 Mar, 77:3, 465-70

13.- Egwuatu Ve; Ezeh Io. Vaginal delivery in Nigerian women after a previous cesarean section. Int J Gynaecol Obstet, 1990 May, $32: 1,1-6$

14.- Rosen Mg; Dickinson Jc. Vaginal birth after cesarean: a metaanalysis of indicators for success. Obstet Gynecol, 1990 Nov, 76:5 Pt 1, 865-9

15.- Coltart Tm; Davies Ja; Katesmark M. Outcome of a second pregnancy after a previous elective caesarean section. Br J Obstet Gynaecol, 1990 Dec, 97:12, 1140-3

16.- Sanchez Ramos L; Kaunitz Am; Peterson Hb; Martinez Schnell B; Thompson $\mathrm{Rj}$. Reducing cesarean sections at a teaching hospital. Am J Obstet Gynecol, 1990 Sep, 163:3, 1081-7; discussion 1087-8.

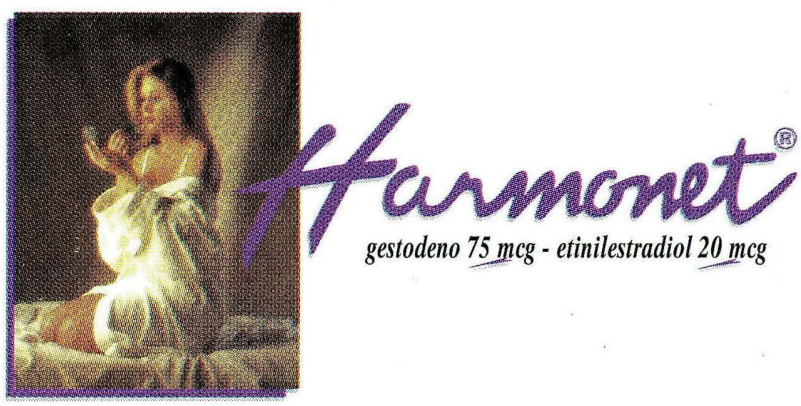

FORMULA

Cada gragea contiene: Gestodeno $75 \mathrm{mcg}$, Etinilestradiol $20 \mathrm{mcg}$, Lactosa $37,155 \mathrm{mg}$, azúcar $19,66 \mathrm{mg}$ almidón de maiz $15,5 \mathrm{mg}$, carbonato de calcio $8,697 \mathrm{mg}$, talco $4,242 \mathrm{mg}$ polietilenglicol $60002,18 \mathrm{mg}$, povidona K-25 1,7 $\mathrm{mg}$, estearato de magnesio $550 \mathrm{mcg}$, povidona K-90 $171 \mathrm{mcg}$, cera E $50 \mathrm{mcg}$.

INDICACIONES

Harmonet está indicado para la prevención del embarazo en mujeres que deciden utilizar este método. POSOLOGIA Y FORMA DE ADMINISTRACION

Para lograr una eficacia anticonceptiva óptima, HARMONET deberá ingerirse diariamente según las indicaciones y con intervalos diarios que no excedan las 24 horas. Debe instruirse a la paciente para que tome las grageas todos los días a la misma hora, preferiblemente con la comida de la noche 0 bien antes de acostarse. CONTRAINDICACIONES

Harmonet está contraindicado en las mujeres que presenten:

Antedentes o diagnóstico de trastornos trombóticos arteriales o venosos, trastornos embólicos o situaciones que pudieran predisponer a la paciente (por ejemplo, defectos de la coagulación, valvulopatías cardiacas, fibrilación auricular); transtornos agudos o crónicos de la función hepática (incluyéndose entre éstos el síndrome de DubinJohnson 0 al síndrome de Rotor), antecedentes o diagnóstico del tumores hepáticos antecedentes de ictericia idiopática o de prurito importante durante el embarazo; antecedentes o diagnóstico conocido o sospechado de neoplasias hormonodependientes ( por ejemplo, cancer de mama o de endometrio); trastornos del metabolismo lipídico; anemia falciforme; diabetes mellitus con alteraciones vasculares; antecedentes de herpes gestacional; otosclerosis que se agravan durante el embarazo; sangrado vaginal no diagnósticado; embarazo conocido o sospechado; hipersensibilidad a cualquiera de los componentes de Harmonet.

\section{EFECTOS SECUNDARIOS}

Los siguientes efectos secundarios se han registrado en un total de 1338 pacientes que recibieron Harmonet durante el curso de tres estudios clínicos:

Generales Incidencia $>3 \%$ : dolor abdominal, dolor de espalda, dolor en los genitales, dolor pélvico. Incidencia < que 1\%: distensión del abdomen, abcesos, reacciones alérgicas, astenia, fiebre, síndrome gripal, pesadez en las extremidades, infecciones, malestar, moniliasis, artritis reumatoidea. Sistema Cardiovascular Incidencia $>3 \%$ : migraña. Incidencia de 1\%-3\%: venas varicosas. Incidencia < $1 \%$; dolor al pecho trombosis de las venas profundas sofocos, hipertensión, palpitaciones, taquicardia, tromboembolia, tromboflebitis, vasodilatación, y otros transtornos cardiovasculares. Sistema Digestivo Incidencia $<3 \%$ : Náuseas. Incidencia $1 \%-3 \%$ : Vómito. Incidencia < $\%$ : colecistis, diarrea, flatulencia, transtornos vesiculares,gastritis, gastroenteritis, transtorno gastrointestinales, hepatopatía, aumento del apetito estomatitis. Sistema Metabólico Incidencia < $1 \%$ edema aumento o pérdida del peso. Sistema Nervioso Incidencia $>3 \%$ : Cefaleas, nerviosismo. Incidencia 1\%-3\%: Depresión mareos, alteraciones en la libido. Incidencia $<1 \%$ : amnesia, ataxia, hostilidad, parestesia, transtorno en el sueño, somnolencia, sudoración excesiva. Sistema Respiratorio Incidencia < $1 \%$ : bronquitis, faringitis, rinitis, sinusitis. Dermatológicos. Incidencia $>3 \%$ : Acné. Indicidencia < $1 \%$ : alopecía, cloasma, eczema, prurito, eritema otros trastornos dermatológicos. Organos de los sentidos. Incidencia < $1 \%$; visión anormal, tinnitus, sordera tota transitoria. Sistema Urogenital. Incidencia $>3 \%$; amenorrea, sangrando intermestrual, goteo, tensión mamaria. Incidencia < $1 \%$; cistitis, d ismenorrea, disuria, dolor en los genitales, galactorrea, leucorrea, nefritis, quistes ováricos, Incidencia $<1 \%$ : cistitits, dismenorrea, disuria, dolor en los genitales, galactorrea, leucorrea, nefritis, quistes ovaricos, cálculos renales infecciones del trato urinario, sequedad vaginal, moniliasis vaginal, vaginitis, transtornos vulvovaginales. Por otra parte, los siguientes efectos secundarios se han registrado en pacientes que recibian anticonceptivos orales y se consideraron relacionados con la droga administrada:Náuseas, Vomitos, sintomas gastrointestinales, (Tales como cólicos abdominales e inflamacion), sangrado intermenstrual, goteo, alteraciones en el flujo menstrual, amenorrea, infertilidad temporaria con posterioridad a la descontinuación del tratamiento, edema, melasma que puede persistir, aumento o disminución de peso, alteraciones en la erosión y secreción cervical, disminución en el volumen de leche en el postparto inmediato, ictericia colestatica, migraña, eritema (alergico), depresion mental, menor tolerancia a los carbohidratos, candidiasis vaginal, acentuacion de la curvatura de la cornea, intolerancia a los lentes de contacto, cambio en las mamas: tensión, aumento de tamaño, secreción. Existen evidencias de una posible relación entre los siguientes efectos secundanios y la úlización de anticonceptivos orales, aunque aun debe conficior Los siguien nes efoct asociacion no ha sido acn conflios se han rection, neuritis oplica, cambios en el apelito, sindrome similar a la cistli, cefarda del cabello, cha sindrome uremico hemoltuco, sindrome de BuddChiari, acne, cambios en la lidilo. collis, enternedad cerebrovascular con prolapso de la valunla mitral, sindrome similares a lupus.

Para mayor información, véanse las advertencias y precauciones.

SOBREDOSIS

La sobredosis puede causar náuseas o vómitos; en las mujeres puede producirse sangrado por supresión. En niños, no se han registrado efectos graves después de la utilización de altas dosis de anticonceptivos orales. PRESENTACION

Envase con 21 grageas. Registro INVIMA 006606

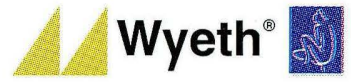

Lider mundial en el cuidado de la salud femenina 